\title{
In memoriam Eduard Eicher
}

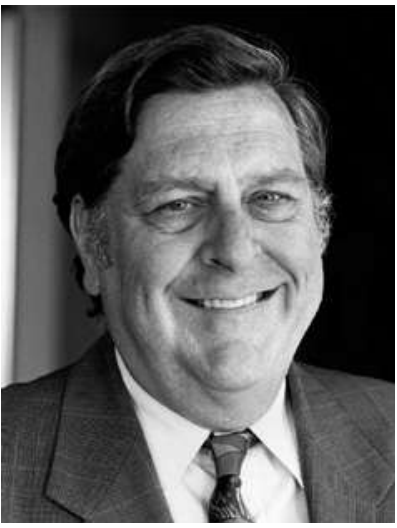

Dr. med. Eduard Eicher 1934-2015

Dr. med. Eduard Eicher, Facharzt für Chirurgie und Handchirurgie, gehörte dem Zentralvorstand der FMH von 1984-2000, also während 16 Jahren, an. Er war vorher in manchen Chargen in der Medizinischen Gesellschaft Basel, zuletzt als Präsident, tätig.

Er wurde als Nachfolger von Dr. R. Tröster in den ZV gewählt (damals galt noch die ungeschriebene Regelung, dass alle fünf Universitätskantone mit medizinischen Fakultäten im ZV vertreten sein sollten). Sehr bald setzte sich Dr. Eicher intensiv und nachhaltig für die Qualitätsförderung und -sicherung in der Medizin ein. Er entwickelte viele theoretische und praktische Konzepte, präsidierte manche FMH-Kommissionen und eignete sich auch dank seiner mannigfaltigen internationalen Kontakte ein grosses Wissen auf diesem Gebiet an. Er war ein aktiver Befürworter von gesamtschweizerischen QualitätssicherungsProgrammen. Zu seinen grundsätzlichen und oft sehr

\section{Eduard Eicher war ein aktiver Befürworter von gesamtschweizerischen Qualitätssicherungs- Programmen.}

zeitaufwendigen Beschäftigungen gehörte auch die Entwicklung und Förderung von Guidelines, die er zusammen mit vielen medizinischen Fach- und kantonalen Ärztegesellschaften zu entwickeln versuchte. Mit diesen Argumentationen setzte er sich auch sehr früh für die Zertifizierung von Operationssälen ein, insbesondere derjenigen in privaten chirurgisch tätigen Arztpraxen.
Dr. Eicher war viele Jahre in Basel in einer Gruppenpraxis tätig und erkannte sehr früh, dass die ärztliche Tätigkeit nur möglich ist auf einer soliden ökonomischen Basis. Deshalb war er von Anfang an in der Arbeitsgruppe der FMH tätig, welche die Grundlagen für einen gesamtschweizerischen Arzttarif erarbeiten sollte (GRAT). Diese Arbeiten führten in der Folge zum TARMED, der heute als Abrechnungs- und Verhandlungsgrundlage für die Ärzteschaft dient.

Die ruhige und überlegte Art von Dr. Eicher führte auch dazu, dass er als FMH-Vertreter im In- und Ausland eine sehr angesehene Person war. Er wurde deshalb unter anderem auch in die Leitungsgremien der Europäischen Spitalarztvereinigung (AEMH) und der Schweizerischen Ärzte-Krankenkasse berufen. Er hat massgeblich das positive Bild, das die FMH im Ausland geniesst, mitgeprägt.

Dr. Eicher war auf eine ungezwungene Art ein Freund der Natur. Er war ein leidenschaftlicher Fischer im nahen Elsass oder im Kanton Bern, wo er sehr oft seine Freizeit mit Freunden und natürlich seinem Berner

\section{Eduard Eicher war im besten Sinne des Wortes} ein kollegialer Typ.

Sennenhund verbrachte. Daneben betreute er mit viel Sorgfalt und Wissen seine Bienenvölker und erfreute sich immer wieder an deren gutem Honig.

Am wohlsten fühlte sich Eduard Eicher aber im Kreise seiner Familie. Tief traf ihn vor 14 Monaten deshalb die schwere Erkrankung und der Tod seiner Ehefrau, mit der er über 50 Jahre verheiratet war. Nur wenige Wochen nach ihrem Hinschied bekam er selbst die Diagnose einer heimtückischen Erkrankung, die er trotz Zuversicht und Hoffnung nicht besiegen konnte.

Dr. Eicher war im besten Sinne des Wortes ein kollegialer Typ. Er hatte ein offenes und feines, ehrliches und vornehmes Wesen. Wir werden ihn in allerbester Erinnerung behalten.

Dr. med. René Salzberg, Basel, Mitglied des Zentralvorstandes FMH 1981-2000 